\title{
THE DIAGNOSIS OF CORRECTED TRANSPOSITION OF THE GREAT VESSELS
}

\author{
BY \\ GEOFFREY H. WATSON \\ From the Royal Manchester Children's Hospital, Manchester, and Department of Child Health, University \\ of Manchester
}

Received October 14, 1963

Many reports on corrected transposition of the great vessels have appeared since the classical article by Anderson, Lillehei, and Lester in 1957, and the features of the condition are now well known. Several authors have emphasized the value of venous, and more recently selective, angiocardiography in establishing its presence (Morgan et al., 1962, with earlier references). The purpose of this paper, which is based on 8 cases seen during their childhood, is to discuss the value of selective cine-angiocardiography in corrected transposition with particular reference to the detailed diagnosis of the other cardiac anomalies commonly present, and to mention a few minor features that might play a part in diagnosis.

The diagnosis of corrected transposition in these children was made on the basis of clear angiocardiographic evidence. Additional defects were present in all: one child, the only one free from symptoms, had a mild pulmonary valvular stenosis alone; 4 had ventricular septal defects with moderate or large left-to-right shunts and severe pulmonary hypertension (presumptive in one); and 3 had severe pulmonary stenosis and ventricular septal defects which in 2 were large, so giving a tetralogy-like picture. All 8 had a ventricular septum. In addition some features of 4 cases of single ventricle with a similar arrangement of the great vessels are mentioned.

Physical examination was usually, as others have found, of no particular diagnostic help; in particular, convincing signs of a left atrio-ventricular valve lesion were detected in only one. Chest radiographs, however, usually showed features suggestive of corrected transposition of the great vessels as described by others (Anderson et al., 1957; Schiebler et al., 1961). In addition a mild-tomoderate degree of dextroversion was seen in 3 cases; the presence of even a mild degree of dextroversion should bring to mind the possibility of corrected transposition (Engle et al., 1960). A radiological feature seen in two cases with pulmonary hypertension but not previously reported was the presence on the upper right mediastinal border of a prominence (Fig. 1) caused by great enlargement of the medially-placed pulmonary artery.

A characteristic electrocardiographic feature is absence of $Q$ from leads V5 and V6, and this was found in all the cases. In two of them small q waves were present in V7, but these children had some dextroversion and V7 probably faced the left ventricular cavity. (In this paper the ventricle receiving blood from the right or left atrium is referred to as the right or left ventricle respectively.) Heart block of various degrees was present in 5 cases. Schiebler et al. (1961) said that signs of heart block became more obvious with increasing age, and this sequence was seen in one. In two, however, the duration of the P-R interval, which in infancy had been abnormally long, remained unchanged on several recordings made in later childhood so that it then fell within the upper limit of the normal range. Only 2 of the 4 children with single ventricle and great vessels similar in arrangement to that found in corrected transposition had the typical electrocardiographic pattern. 


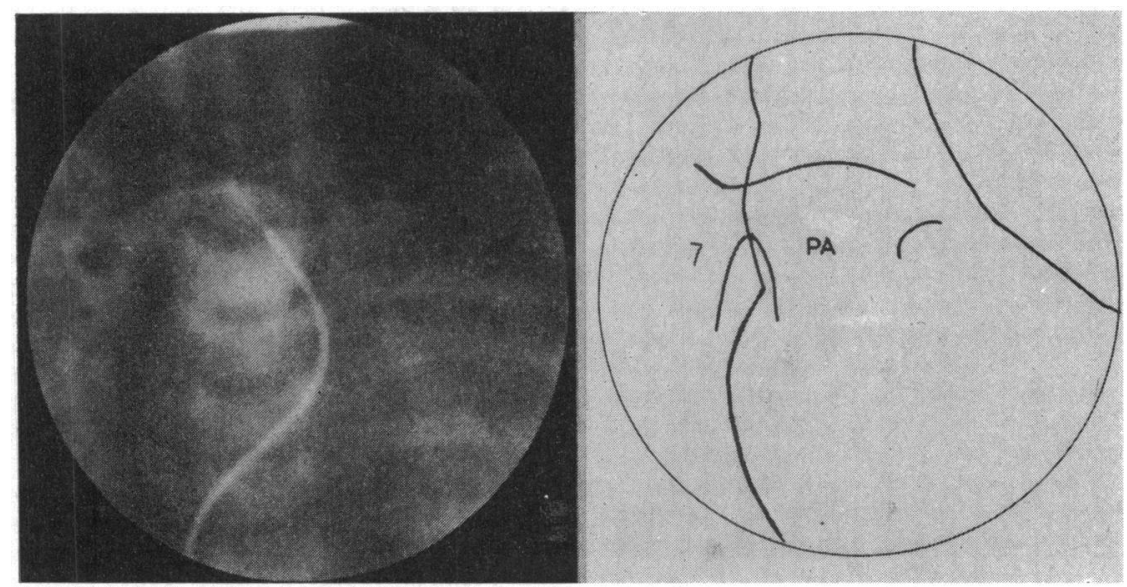

FIG. 1.-Injection into large pulmonary trunk (PA) in case of corrected transposition with large ventricular septal defect and left-to-right shunt. Pulmonary artery caused prominence on right border of mediastimun.

Cardiac catheterization was carried out in all cases, and, in general, the findings were in accordance with the experience of others. Entry into the right ventricle was always achieved but was difficult in several cases, not only because of the cephalic position of the right atrio-ventricular valve noted by Schiebler et al. (1961) but also bzcause, in some, the catheter had to be directed unusually anteriorly even in the absence of dextroversion. In addition to the ventricular septal defect or pulmonary stenosis or both shown by the hæmodynamic findings, the evidence available towards the end of catheterization had in all cases led to the suspicion, at least, of the presence of some other anomaly, but in only one case was the diagnosis of corrected transposition felt to be firmly established at this stage of investigation, though in 4 more it was thought to be probable.

Selective cine-angiocardiography was performed in each child and the films were developed and seen, though not projected, at once. Injections were made into the right ventricle in the posteroanterior position and in most of them in the lateral position as well; and in some, injections were also made into the left ventricle, aorta, or pulmonary artery. The nature of the diagnostic problem determined the sequence of the injections, which were followed by no adverse effects.

Injections into the right ventricle in the frontal position were the most informative and showed the typically medial position of the pulmonary trunk. The "tail" of the right ventricle extending towards the left (Lester et al., 1960) was seen in cases without dextroversion (Fig. 2 and 6). The diagnosis of corrected transposition of the great vessels, however, should not rest on these features alone, for a rather similar picture may occasionally be seen in cases with mild dextroversion but normal relation of the great vessels (Fig. 3). Ventricular inversion is always present in corrected transposition except in some of the cases with marked dextroversion (Shaher, 1963), but the arrangement of the columnæ carneæ and papillary muscles is often not clear enough to be diagnostic. An upward prominence of the right ventricle above the right atrio-ventricular valve and in front of the pulmonary trunk was seen in 4 children (Fig. 2, 4, 7, 8, 9, and 10) particularly in the lateral view, and as it has not been seen in other conditions it may help to confirm the presence of inversion of the ventricles. It is nevertheless advisable for the position of the aorta to be seen as well.

The origin of the aorta was always seen after an injection into the right ventricle either as a result of a right-to-left shunt across a ventricular septal defect or after the passage of the medium through the lungs: the ascending aorta usually lay to the left of and anterior to the pulmonary trunk, but in one case lay directly anterior to it. In some cases with a large left-to-right shunt the aortic root was not easy to see, and the demonstration of details required another injection made in the vicinity of the ventricular septal defect or into the left ventricular outflow tract when acces- 


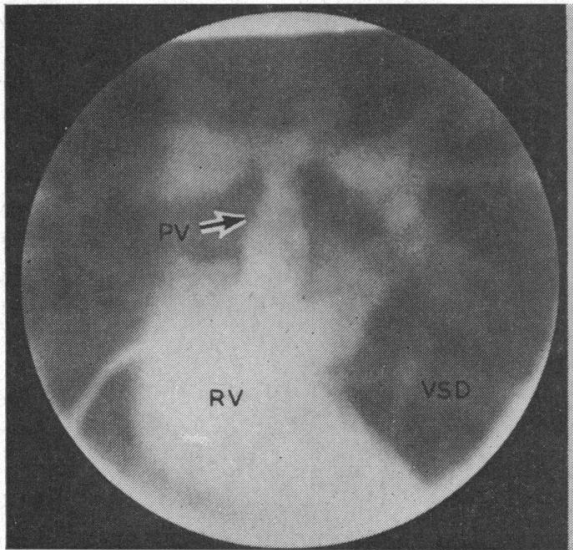

Fig. 2.-Injection into right ventricle (RV) in case of corrected transposition; valvular pulmonary stenosis (PV), hypoplasia of pulmonary artery, right-to-left shunt through ventricular septal defect (VSD), and "tail" of right ventricle extending to left.

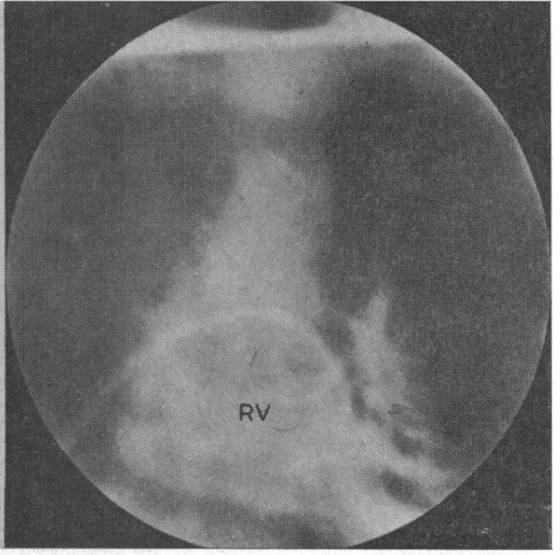

FIG. 3.-Isolated pulmonary valvular stenosis proved at operation. Right ventricle of somewhat similar shape.

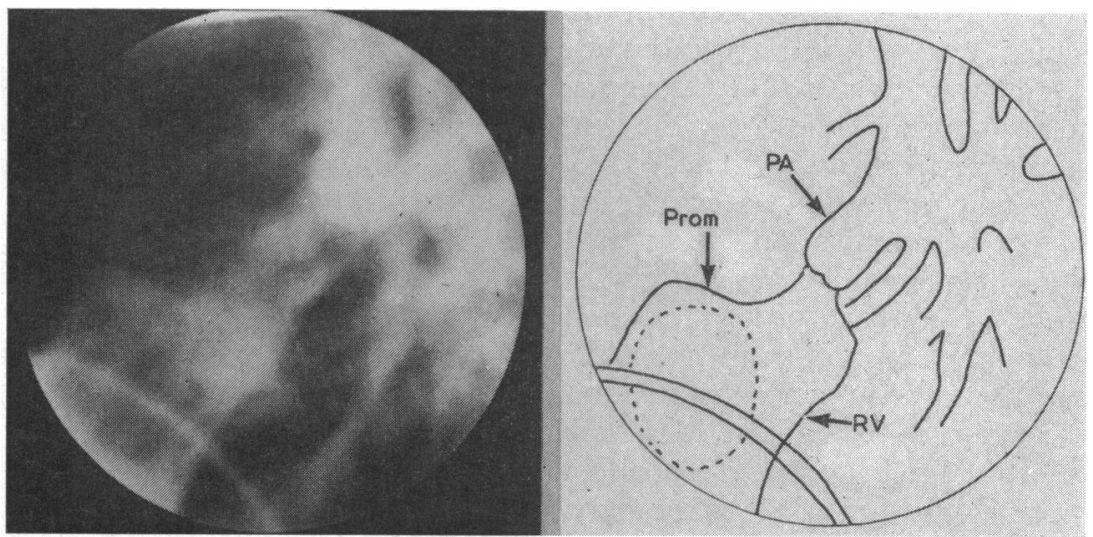

FIG. 4.-Same case as Fig. 2; lateral view showing prominence (prom) of right ventricle over right atrio-ventricular valve.

sible (Fig. 5 and 6). As Lester et al. (1960) and Morgan et al. (1962) have found, lateral views have as a rule provided no additional diagnostic information concerning the presence of corrected transposition, which has always been more obvious on the frontal view, though of course other views may be indicated as well in order to show other features.

When the pulmonary trunk arises from the right ventricle in the normal way the pulmonary valve often faces more forwards than downwards, and the cusps are usually, and the outflow tract often, seen better in a lateral view (or in the tetralogy in a right anterior oblique view) than in the frontal view. In corrected transposition the pulmonary trunk is so posterior that it is more vertical and the valve and subvalvar region are usually well seen in the postero-anterior view (Fig. 2, 5, 6, 8, and 10).

It was possible to introduce the catheter into the pulmonary trunk in 7 of the 8 cases. Pressure tracings made during withdrawal into the right ventricle were typical of valvular stenosis in 4 , but 

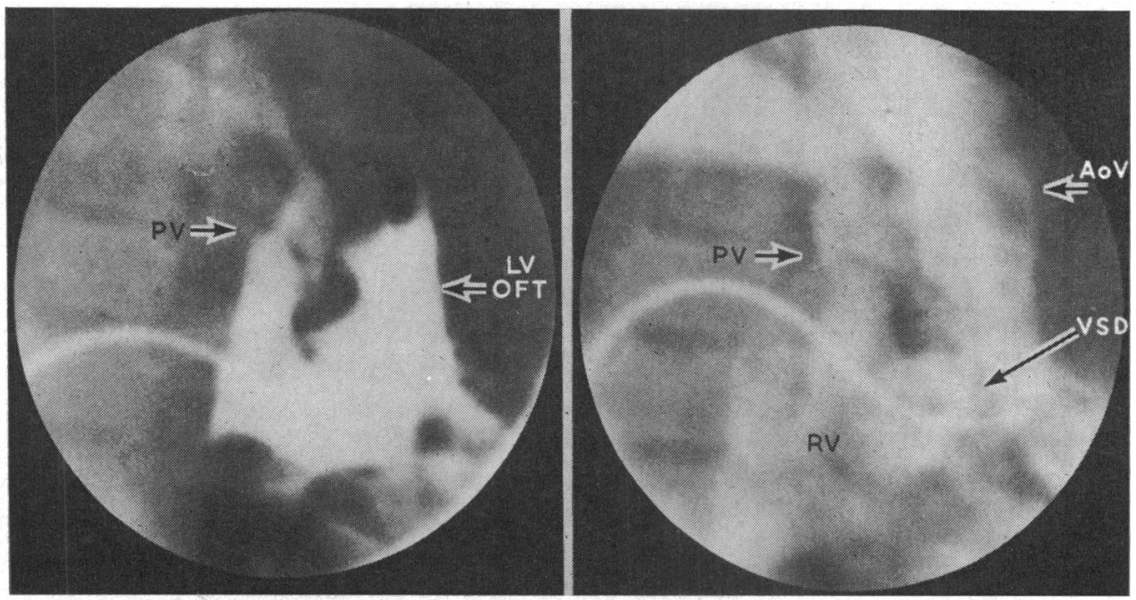

FIG. 5 and 6.-Early and late stages of injection showing valvular pulmonary stenosis and size of ventricular septal defect in similar case. LVOFT=left ventricular out-flow tract leading to aortic valve (AoV).

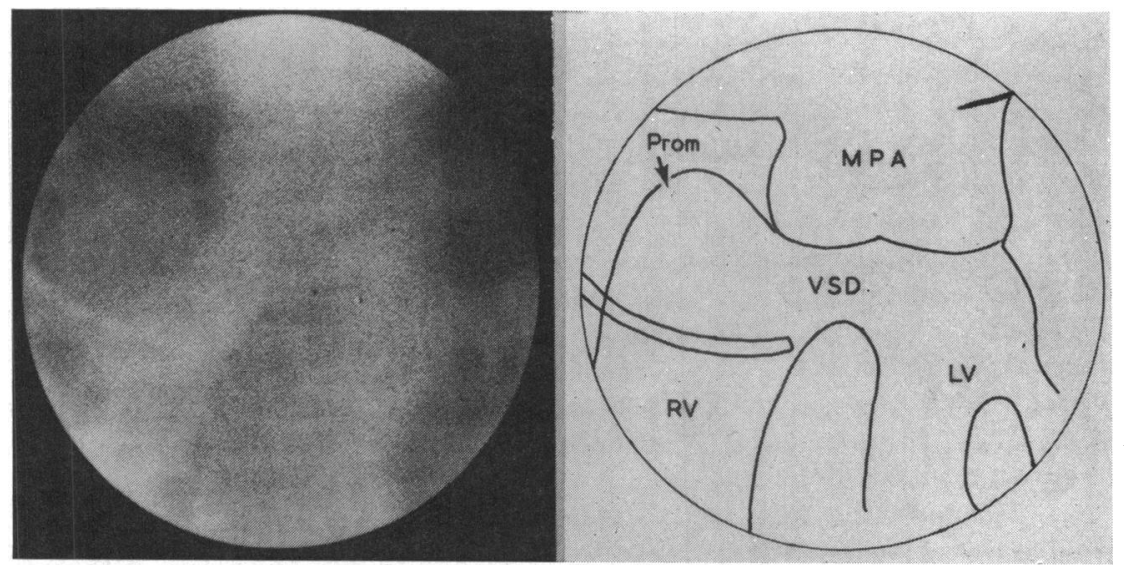

Fig. 7.-Injection into ventricular septal defect (catheter has recoiled into right ventricle) in case of corrected transposition with severe pulmonary hypertension and left-to-right shunt. Pulmonary artery appears to override defect.

in one of these the angiograms and necropsy showed sub-valvular stenosis alone (Fig. 10), while another had valvular stenosis and severe systolic narrowing of the subvalvar region. This misleading feature was in part attributable to the shortness of the subvalvar stenosis and its close proximity to the valve, but was largely due to the instability of the catheter when its tip lay just above the pulmonary valve, from which position it tended to flip out suddenly into the right ventricle. Hypoplasia of the pulmonary artery and valve and subvalvar and combined stenoses are common in the reported cases and, in view of the surgical difficulties they present, 'great care must be taken before deciding that a pulmonary stenosis is suitable for operation. These anomalies can only be demonstrated by angiography.

Information about the position and size of the ventricular septal defect may be useful from the surgical view-point: presumably a small defect in the anterior part of the septum would offer a better surgical risk than a more posterior defect such as that shown in Fig. 7, as access would be easier and damage to the conducting tissues and pulmonary valve less likely. The position and 
size of the ventricular septal defect may be suggested by the path of the catheter and the ease with which it enters the aorta, but selective angiocardiograms made into the right ventricle provide more, and more certain, information in cases with a right-to-left shunt (Fig. 2). An injection into the region of the septal defect provides a better demonstration (Fig. 5 and 6) and of course it is best to inject into the defect or the left ventricle, if accessible, in cases where there is only a left-to-right shunt (Fig. 7). In cases of ventricular septal defect with a normal relation of the great vessels the defect is best shown in a left anterior oblique view (Sones, 1958), but in corrected transposition the septum may lie in a more antero-posterior place, particularly if there is some degree of dextroversion. The frontal view is therefore often satisfactory for demonstrating a ventricular septal defect unless there is gross dextroversion. The ventricular septum may not be seen quite end-on, but this may make it easier to decide where the defect lies.

A very large ventricular septal defect with bi-directional shunts may be hard to distinguish from a single ventricle in which there may be incomplete mixing of oxygenated and unoxygenated blood,
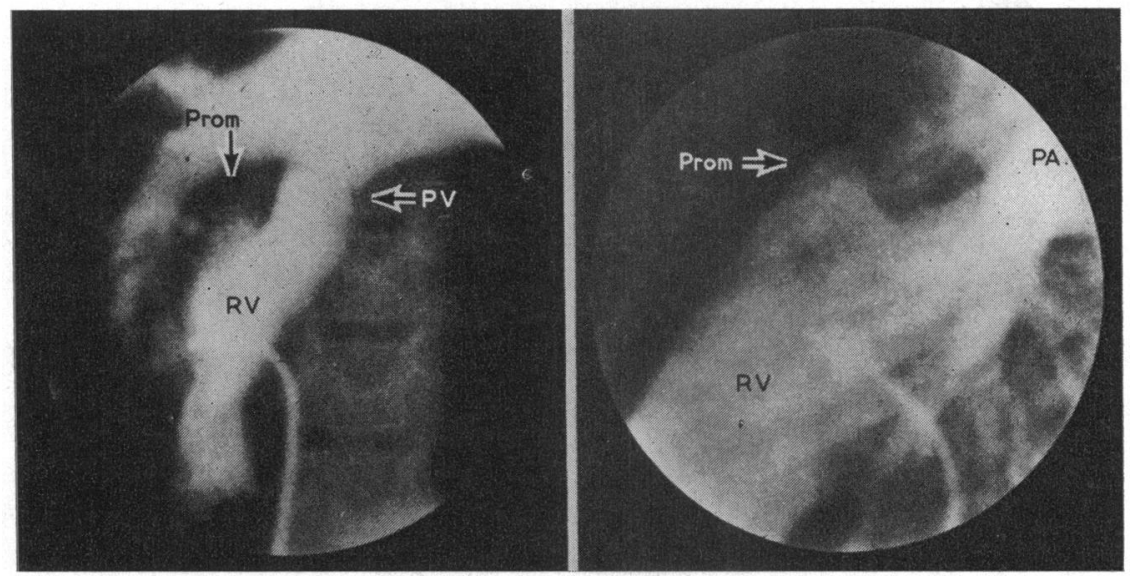

Fig. 8 and 9.-Case of corrected transposition with mild pulmonary valvular stenosis. Injection into right ventricle (frontal and lateral views) showing prominence of right ventricle over right atrio-ventricular valve.

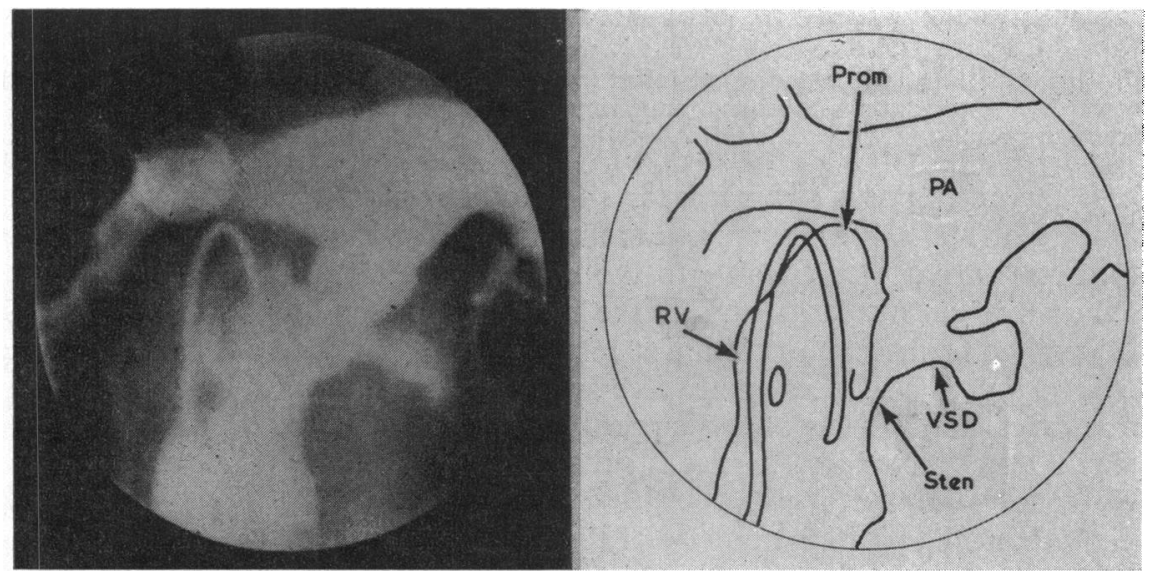

Fig. 10.-Corrected transposition, small ventricular septal defect, severe subvalvar pulmonary stenosis. Right ventricular injection. 


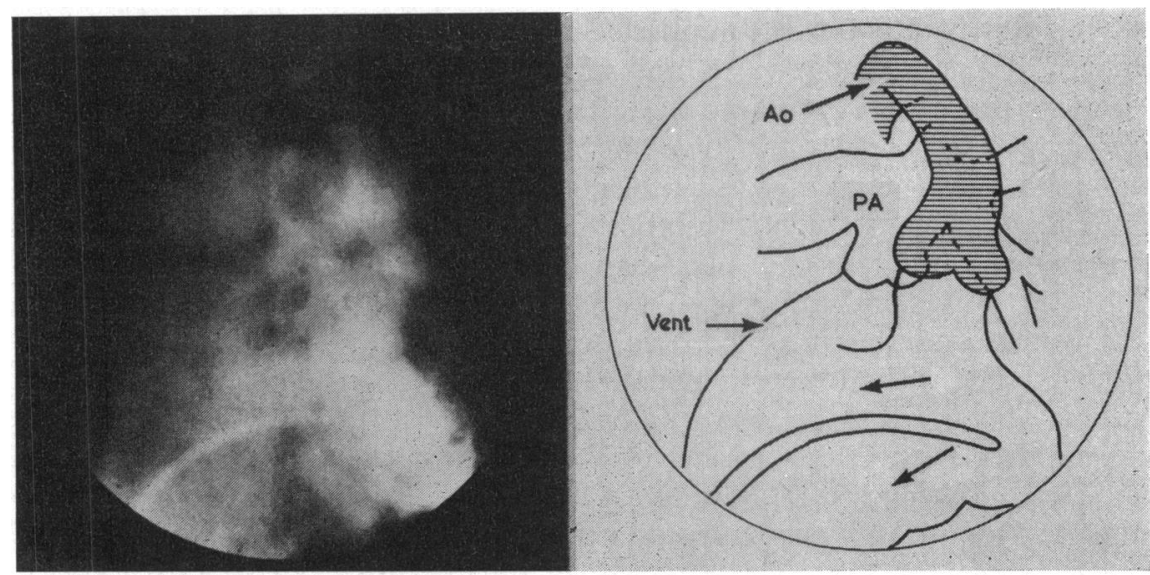

FIG. 11.-Single ventricle with arrangement of great vessels similar to that seen in corrected transposition. Injection into left side of ventricle; medium spreads on a wide "front" throughout ventricle (arrows).

and this difficulty is particularly likely to be encountered in cases of corrected transposition, where the defects tend to be large. The origin of the great vessels in 45 per cent of cases of single ventricle is similar in arrangement to that characteristic of corrected transposition (Anderson, 1960) and, even if one of the great vessels is entered, catheterization alone may not distinguish between a single ventricle of this type and corrected transposition with a large ventricular septal defect. Angiocardiograms made after injections into various sites in the ventricle and in more than one projection can provide good evidence for or against the presence of any sizeable ventricular septum (Fig. 11) or may show a small outflow chamber. The appearances of such a chamber must be distinguished from a similar picture which can be produced if medium streams from the right ventricle into the left ventricular outflow tract without entering the lower part of the left ventricle (Fig. 2 and 5).

Injections into the left atrium, when accessible, may occasionally provide diagnostic information in this situation, and have done so in two cases of single ventricle, by showing the immediate passage of medium into the same ventricular chamber as that entered from the right atrium. In addition, of course, such injections give some information about the competence (or even the existence) of the left atrio-ventricular valve and the nature of any atrial septal defect, though the frontal view will not usually be optimal for demonstrating a defect (Sones, 1958).

Although ventricular septal defects in corrected transposition are usually large and easily recognized during cardiac catheterization, the frequency with which these two anomalies are associated is so high that particular care is required before declaring the ventricular septum to be intact. The value of right ventricular selective angiocardiography in demonstrating a small ventricular septal defect is shown in Fig. 10. In this boy, who had sub-pulmonary stenosis, the right ventricular systolic pressure was $180 \mathrm{~mm}$. $\mathrm{Hg}$, but oxygen saturations did not provide conclusive evidence of any shunt. Such a small defect might be missed at operation though its closure would clearly be advisable.

An abnormality of the left atrio-ventricular valve is common though it is more frequently reported post mortem than in clinical cases. Its presence may be suggested by the physical or hæmodynamic findings or, less conclusively, by radiological or angiocardiographic evidence of left atrial enlargement, and selective left ventricular angiocardiography may provide more direct evidence of incompetence. Fairly severe regurgitation was demonstrated in this way in the one child in this series who had clinical evidence of it. However, in those cases in which the ventricular septal defect allowed entry into the left ventricular outflow tract it was not possible to 
pass the catheter down into the left ventricle far enough for much of the injected material to approach the left atrio-ventricular valve, and mild degrees of regurgitation could well have been missed. Injections made after entry into the left ventricle by way of the left atrium or aorta would be more satisfactory in such cases, but have not been made in this series.

In patients with or without corrected transposition who have a large ventricular septal defect with severe pulmonary hypertension it is impossible to exclude the additional presence of a patent ductus by catheterization data alone. If, when this situation is encountered, the catheter can be introduced into the aorta, and especially if it can be passed round the aortic arch, aortography should be performed and the presence or absence of the ductus conveniently shown. Only a small injection is needed with no, or minimal and temporary, hæmodynamic changes so, as re-entry into the aorta may be difficult, the opportunity for aortography, if it is indicated by the available data, should be taken whenever the catheter reaches the appropriate site even if blood sampling has not been completed. This approach, however, is less successful in cases with corrected transposition because of the greater difficulty encountered in manipulating the catheter up the ascending aorta. In this series entry into the aorta was achieved in three, in only one of which was aortography indicated for this purpose: the possibility of a patent ductus was thus excluded. In patients without corrected transposition aortography performed in order to demonstrate a possible patent ductus can be carried out in either the lateral or postero-anterior position. In some cases of corrected transposition the ascending and descending aorta and the pulmonary trunk are largely superimposed in the frontal view and aortography is better performed in the lateial position.

Ascending aortography and immediate examination of the films at a fairly early stage of the investigation have also been useful in cases where the findings were compatible with a truncus arteriosus, the presence of which would have made further detailed investigation unnecessary.

Injections into other sites may occasionally be useful, as in the case of a child with corrected transposition of the great vessels, a large bi-directional shunt across a ventricular septal defect, and severe pulmonary hypertension: the aorta could not be entered, but a small but rapid injection into the greatly dilated pulmonary trunk demonstrated a small patent ductus leading to the descending aorta and so avoided the need for trans-arterial aortography.

\section{SUMmARY AND CONCLUSIONS}

In each of these 8 cases the evidence available at the end of cardiac catheterization, but excluding the information provided by angiocardiography, suggested the presence of a complex lesion. After the investigation it was felt in most cases that the diagnosis of corrected transposition would have been inadequately established without selective angiocardiography; this also added a considerable amount of information some of which could not have been provided by any other investigations, including venous angiocardiography. It may by these means be possible to select certain children with valvular pulmonary stenosis and ventricular septal defects of suitable nature in whom operation may offer a lower risk than that usually associated with corrected transposition of the great vessels. It seems reasonable to conclude that patients with clinical features that are not completely characteristic of the commonly found anomalies but arouse a suspicion of corrected transposition should only undergo cardiac catheterization if selective angiocardiography, preferably with immediate examination of the films, can be performed during the investigation.

This work was mostly carried out during the tenure of a lectureship in Child Health at Manchester University, and I am grateful to Professor W. F. Gaisford and to other physicians for referring cases. I am indebted to Mr. W. Healey, Superintendent Radiographer, for the cine-angiograms.

\section{REFERENCES}

Anderson, R. C. (1960). In Brenneman's Practice of Pediatrics, Chap. 38., Congenital heart disease, p. $70 . \quad$ Prior, Hagerstown, Maryland.

, Lillehei, C. W., and Lester, R. G. (1957). Corrected transposition of the great vessels of the heart. A review of 17 cases. Pediatrics, 20, 626. 
Engle, M. A., Steinberg, I., Lukas, D. S., and Goldberg, H. P. (1960). Spectrum of cardiac anomalies associated with "corrected transposition" of the great vessels. Circulation, 22, 745.

Lester, R. G., Anderson, R. C., Amplatz, K., and Adams, P. (1960). Roentgenologic diagnosis of congenitally corrected transposition of the great vessels. Amer. J. Roentgenol., 83, 985.

Morgan, J., Pitman, R., Goodwin, J. F., Steiner, R. E., and Hollman, A. (1962). Anomalies of the aorta and pulmonary arteries complicating ventricular septal defect. Brit. Heart J., 24, 279.

Schiebler, G. L., Edwards, J. E., Burchell, H. B., DuShane, J. W., Ongley, P. A., and Wood, E. H. (1961). Congenital corrected transposition of the great vessels: a study of 33 cases. Pediatrics, 27, 851 .

Shaher, R. M. (1963). The syndromes of corrected transposition of the great vessels. Brit. Heart J., $25,431$.

Sones, F. M. (1958). Cine-cardio-angiography. Pediat. Clin. N. Amer., 5, 945. 